\title{
Delayed Diagnosis of Tandem Spinal Stenosis: A Retrospective Institutional Review
}

\author{
AMIT BHANDUTIA, MD, LUKE BROWN, MD, MBA, ALYSA NASH, MD, IAN BUSSEY, MD, MARK \\ SHASTI, MD, EUGENE KOH, MD, PHD, KELLEY BANAGAN, MD, STEVEN LUDWIG, MD, DANIEL \\ GELB, MD \\ Department of Orthopaedics, University of Maryland Medical Center, Baltimore, Maryland
}

\begin{abstract}
Background: Tandem spinal stenosis (TSS) is defined as simultaneous spinal stenosis in the cervical, thoracic, and/or lumbar regions and may present with both upper and lower motor neuron symptoms, neurogenic claudication, and gait disturbance. Current literature has focused mainly on the prevalence of TSS and treatment methods, while the incidence of delayed TSS diagnosis is not well defined. The purpose of this study was to determine the incidence of delayed TSS diagnosis at our institution and describe the clinical characteristics commonly observed in their particular presentation.

Methods: Following institutional review board approval, an institutional billing database review was performed for patients who underwent a spinal decompression procedure between 2006 and 2016. Thirty-three patients who underwent decompression on 2 separate spinal regions within 1 year were included for review. Patients with delayed diagnosis of TSS following the first surgery were differentiated from those with preoperative diagnosis of TSS.

Results: TSS requiring surgical decompression occurred in 33 patients, with the incidence being $2.06 \%$ in this cohort. Fifteen patients received a delayed diagnosis after the first surgical decompression $(45 \%)$ and were found to have a longer interval between decompressions $(7.6 \pm 2.1$ months versus $4.01 \pm 3$ months, $P=.0004)$. Patients undergoing lumbar decompression as the initial procedure were more likely to have a delayed diagnosis of TSS ( 8 versus 2 patients, $P=.0200$ ). The most common presentation of delayed TSS was pain and myelopathic symptoms that persisted after decompressive surgery.

Conclusion: TSS should remain within the differential diagnosis for patients at initial presentation of spinal stenosis. In addition, suspicion of TSS should be heightened if preoperative symptoms fail to expectedly improve following decompression even if overt myelopathic signs are not present.
\end{abstract}

Level of Evidence: 4

Other and Special Categories

Keywords: tandem spinal stenosis, lumbar decompression, cervical decompression, spinal stenosis, myelopathy, delayed diagnosis, spine surgery

\section{INTRODUCTION}

Tandem spinal stenosis (TSS) is defined as simultaneous spinal stenosis in the cervical, thoracic, and/or lumbar regions. ${ }^{1,2}$ This clinical entity presents with a mix of both upper and lower motor neuron signs and symptoms. TSS may be implicated in up to $28 \%$ of patients presenting with neurogenic claudication. ${ }^{3}$ Current literature has focused mainly on the radiographic prevalence of TSS. However, since the clinical diagnosis can be difficult, the incidence of a delayed diagnosis of TSS is not well defined. ${ }^{3-5}$ The primary goal of this study was to determine the incidence of patients presenting with tandem stenosis to our institution over a 10-year period and determine the number of patients diagnosed in a delayed fashion to ascertain defining characteristics of their particular presentation.

\section{MATERIALS AND METHODS}

\section{Study Population}

After obtaining institutional Review board IRB approval, our institutional database was queried to identify all cases of patients who underwent decompression in 2 different regions of the spine within 12 months over a 10-year time period between December 2006 and December 2016. The hospital database was queried, and all the patients were treated operatively for spinal stenosis were identified through using the ICD-9 codes pertaining 
Tandem Spinal Stenosis

Table 1. Patient demographics of patients diagnosed with tandem spinal stenosis (TSS).

\begin{tabular}{|c|c|c|c|c|}
\hline Demographics & Total & Preoperative TSS Diagnosis & Postoperative (Delayed) TSS Diagnosis & $P$ Value \\
\hline Number $(\%)$ & 33 & $18(55)$ & $15(45)$ & - \\
\hline Age $(y)$, mean \pm SD & $59.1 \pm 9.2$ & $61.1 \pm 10.4$ & $57.2 \pm 7.2$ & .2114 \\
\hline Sex $(\%$ female $)$ & $17(51.5)$ & $8(44.4)$ & $9(60.0)$ & .4905 \\
\hline BMI $\left(\mathrm{kg} / \mathrm{m}^{2}\right)$, mean $\pm \mathrm{SD}$ & $32.2 \pm 6.2$ & $30.8 \pm 6.5$ & $33.9 \pm 5.6$ & .1485 \\
\hline Current smoker (\%) & 66.7 & 77.8 & 53.3 & .1631 \\
\hline Race & & & & .5380 \\
\hline White, n (\%) & $23(69.7)$ & $13(72.2)$ & $10(66.7)$ & - \\
\hline Black, n (\%) & $9(27.3)$ & $4(22.2)$ & $5(33.3)$ & - \\
\hline Asian, n (\%) & $1(3.0)$ & $1(5.6)$ & $0(0)$ & - \\
\hline $\mathrm{CCI}$, mean $\pm \mathrm{SD}$ & $0.76 \pm 1.1$ & $0.89 \pm 1.1$ & $0.6 \pm 1.1$ & .4546 \\
\hline Mean follow-up (mo) & 21.7 & 19.8 & 24.0 & - \\
\hline
\end{tabular}

Abbreviations: BMI, body mass index; CCI, Charlson Comorbidity Index.

to cervical, thoracic, and lumbar stenosis in addition to cervical and thoracic myelopathy and spondylolisthesis $(336.8,336.9,721.1,721.4,721.41,721.42$, $722.70,722.71,722.72,722.73,723.0,723.7,724.00$, $724.01,724.02,724.03,724.09,737.4,756.12$, and 738.4). The patient's electronic medical records (EMR) were reviewed for study eligibility following specific inclusion and exclusion criteria. We included patients who underwent decompressions of 2 distinct spinal regions (ie, cervical, thoracic, lumbar) within a 1-year time period. We assumed that it would be unlikely for a patient to develop symptomatic stenosis de novo in a different spinal region in less than 1 year following surgery; most likely, the stenosis in the other spinal region was present at the time of the original surgery, whether or not it was recognized at the time. Patients were excluded for any nondegenerative etiology, isolated root level surgery, isthmic spondylolisthesis, or history of previous surgical spinal procedures. EMRs of patients with delayed TSS diagnosis were also queried to determine the nature of the delay in diagnosis.

A total of 33 patients were identified and were stratified into 1 of 2 groups: (1) patients diagnosed with TSS prior to their first surgical decompression and (2) patients diagnosed in a delayed fashion following their first surgical decompression. Patients were additionally categorized according to the order of decompression (initially cervical or thoracic versus initially lumbar).

\section{Data Collection}

All collected data was entered into an electronic spreadsheet (Microsoft Excel, Microsoft Office, Redmond, Washington). We followed the Strengthening the Reporting of Observational Studies in Epidemiology (STROBE) guidelines to enhance the quality and minimize the bias of this observational study. ${ }^{6}$ Data collected consisted of patient demographics such as age, sex, race, smoking status, presenting symptoms, comorbidities, associated Charlson Comorbidity Index (CCI), diagnoses, and dates for index and reoperation procedures.

\section{Statistical Methods}

Statistical software (JMP pro, version 13, SAS Institute Inc, Cary, North Carolina) was employed to aid in statistical analysis. Proportions of dichotomous variables were compared using a 2-tailed Fisher exact test. Logistic regression analysis was used in comparing continuous and categorical variables. Means were compared using a 2-tailed student $t$ test. A $P$ value $<.05$ was used for determining significance in all statistical tests.

\section{RESULTS}

\section{Patient Population}

Thirty-three patients were identified with TSS during the study period. During the 10-year study period, 1603 patients received an elective surgical decompression for cervical, thoracic, or lumbar stenosis at our institution. Therefore, the calculated incidence of tandem stenosis requiring contemporaneous surgical decompression of both segments over a 10 -year time period at our institution was $2.06 \%$.

Patients with TSS had a mean age of $59.1 \pm 9.2$ years at the time of their index procedure and a mean body mass index (BMI) of $32.2 \pm 6.2 \mathrm{~kg} / \mathrm{m}^{2}$; $51.5 \%$ were female, $69.7 \%$ Caucasian (23 patients), $27.3 \%$ African American $(\mathrm{n}=9)$, and 1 Asian. The mean number of comorbidities was $2.9 \pm 1.9$ with an associated mean CCI of $0.76 \pm 1.1$. Current smokers made up $66.7 \%$ of our patient population. 
Table 2. Operative characteristics for subgroups.

\begin{tabular}{|c|c|c|c|c|}
\hline Operative Characteristics & $\begin{array}{c}\text { Total } \\
(\mathrm{n}=33)\end{array}$ & $\begin{array}{c}\text { Preoperative TSS } \\
\text { Diagnosis }(\mathrm{n}=\mathbf{1 8})\end{array}$ & $\begin{array}{l}\text { Postoperative (Delayed) } \\
\text { TSS Diagnosis }(\mathrm{n}=15)\end{array}$ & $P$ Value \\
\hline Time between decompressions (mo), mean \pm SD & $5.36 \pm 3.2$ & $4.0 \pm 3$ & $7.6 \pm 2.1$ & .0004 \\
\hline Cervical $>$ distal decompression ${ }^{\mathrm{a}}, \mathrm{n}(\%)$ & $21(63.6)$ & $15(83.3)$ & $6(40)$ & .0143 \\
\hline Lumbar $>$ cranial decompression ${ }^{\mathrm{b}}, \mathrm{n}(\%)$ & $10(30.3)$ & $2(11.1)$ & $8(53.3)$ & .02 \\
\hline Reoperations, n (\%) & $4(12.1)$ & $1(5.6)$ & $3(20)$ & .3083 \\
\hline
\end{tabular}

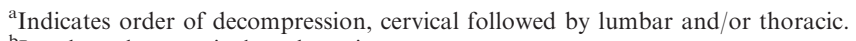

${ }^{\mathrm{b}}$ Lumbar, then cervical or thoracic.

Abbreviation: TSS, tandem spinal stenosis.

Patient demographic information is shown in Table 1.

Of the 33 patients with TSS, 15 patients $(45 \%)$ were diagnosed in a delayed fashion following the first surgical intervention, whereas 18 patients $(55 \%)$ were diagnosed preoperatively. Of the 2 groups, there were no differences in age, gender, BMI, smoking status, race, or comorbidities (Table 1). Of the patients with delayed diagnosis, 9 of 15 patients $(60 \%)$ had persistent preoperative symptoms (pain and myelopathic symptoms) that did not improve with decompressive surgery, prompting additional work-up. The remaining 6 patients $(40 \%)$ were diagnosed due to new onset symptoms, with either new radiculopathy $(n=4)$ or gait and coordination difficulties $(n=2)$ that were not noted by the patient or physician prior to first surgical decompression.

Time between decompressive surgeries overall averaged $5.6 \pm 3.2$ months with a median time of 5.8 months. Average time between decompressive surgeries was 7.6 months with a median of 7 months in patients with a delayed diagnosis of TSS and 4.01 months with a median of 3.32 months in patients

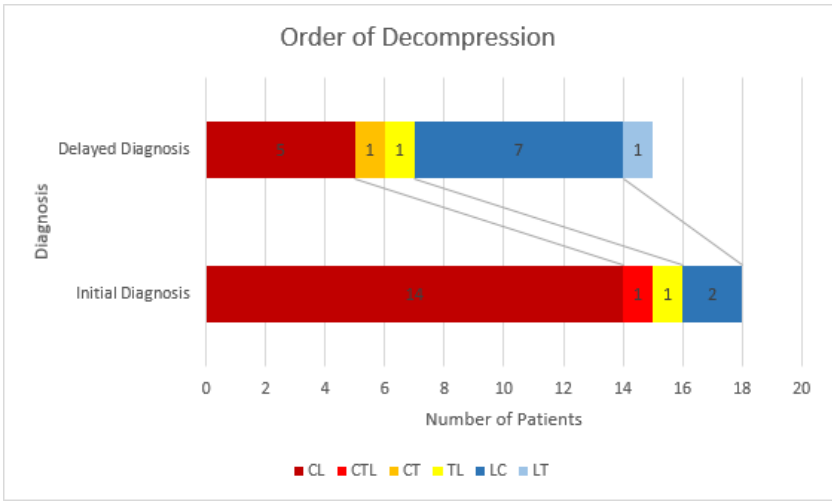

Figure 1. Order of decompression. Patients were substratified into order of decompression (eg, CL refers to patients who first underwent cervical decompression and later went on to receive lumbar decompression; 1 patient received a cervical decompression followed by simultaneous thoracic and lumbar decompression, denoted CITL). given a diagnosis of TSS preoperatively (Table 2). The difference between the groups was significant $(P$ $<.0004)$.

Patients were stratified according to the order of decompression (Figure 1) with 63.6\% $(\mathrm{n}=21)$ undergoing a cervical decompression first, $30.3 \%$ (n $=10)$ lumbar first, and $6.1 \%(\mathrm{n}=2)$ thoracic first. One patient was diagnosed with triple stenosis, having undergone anterior cervical decompression and fusion as the initial procedure and simultaneous decompression and fusion of thoracic and lumbar spine 4.6 months later and was included in the cervical and then lumbar decompression subgroup for analysis. In the group diagnosed with tandem stenosis preoperatively, $83.3 \%(n=15)$ underwent cervical decompression before lumbar decompression, and $53.3 \%(\mathrm{n}=8)$ of patients in the delayed diagnosis group had lumbar decompression performed prior to a more cranial decompression (cervical/thoracic). This was statistically significantly greater than the group diagnosed before surgery ( 2 versus 8 patients, $P=.0200$ ). Of these 8 patients, 6 presented with persistent preoperative symptoms that did not improve with lumbar decompression, prompting additional work-up. Two patients presented with new symptoms that were not present before initial surgery. Additional presenting characteristics following lumbar decompression included postoperative gait instability $(\mathrm{n}=3)$ and upper extremity myelopathic symptoms consisting of coordination difficulties $(\mathrm{n}=3)$, with 1 patient exhibiting both characteristics as well as Hoffman's sign, which was not present at initial preoperative examination. No patient who underwent treatment of lumbar stenosis as the initial surgical intervention demonstrated discrete new neurologic deficits related to exacerbation of their spinal cord compression, whether or not their tandem stenosis was diagnosed preoperatively or in the delayed cohort. No patient who underwent cervical decompression as a secondary procedure failed to improve after that surgery. 


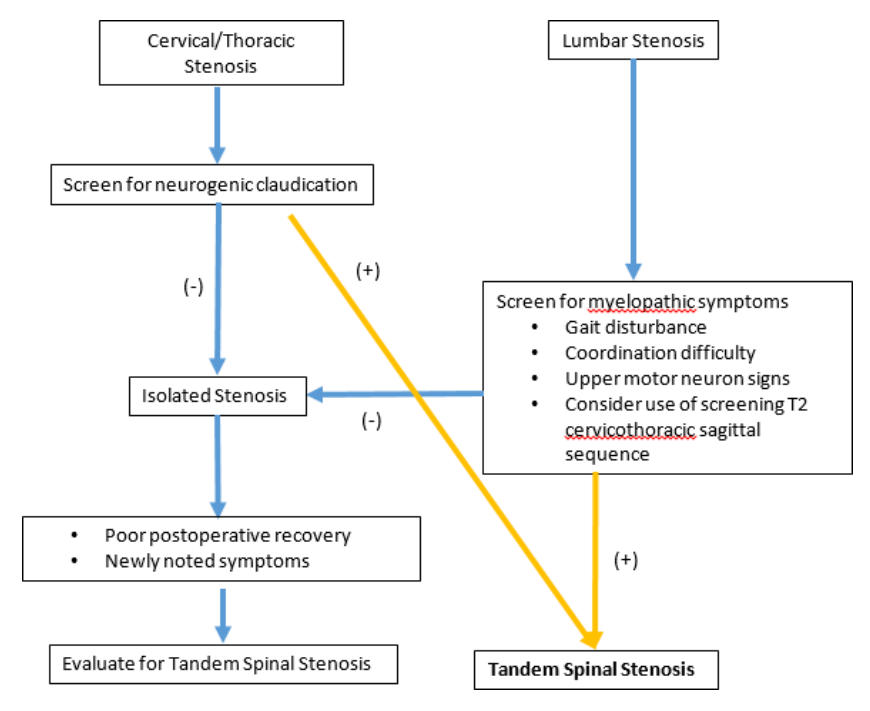

Figure 2. Authors' recommended diagnostic algorithm.

\section{DISCUSSION}

In 1964, Teng and Papatheodorou ${ }^{7}$ discussed combined cervical and lumbar spondylosis in 12 patients. Dagi et $\mathrm{al}^{1,7}$ coined the term "tandem spinal stenosis." In a cadaveric study examining 440 skeletally mature specimens, osseous stenosis in 1 area of the spine predicted osseous stenosis in another area $15 \%$ to $32 \%$ of the time. ${ }^{8}$ Bajwa et $\mathrm{al}^{9-11}$ further noted, in a series of 1072 cadaveric specimens, a prevalence of $1 \%$ for concurrent cervical and thoracic stenosis and $1.42 \%$ for thoracic and lumbar stenosis. Radiographically, Park et $\mathrm{al}^{12,13}$ have shown that $24 \%$ of patients with lumbar stenosis have radiographic thoracic and cervical stenosis with $12 \%$ of patients exhibiting triple stenosis. Seo et $\mathrm{al}^{14}$ demonstrated radiographic tandem steonosis in 142 out of 2113 patients $(6.6 \%)$ with screening of thoracic and cervical regions in patients with lumbar stenosis; 7 of these patients required surgery. In a cross-sectional, observational study of 78 patients diagnosed with symptomatic lumbar spinal stenosis, a predisposition to both radiographic and symptomatic cervical stenosis was present in the experimental group when compared to an age-matched and sex-matched cohort. $^{15}$

In the current cohort, the incidence of tandem stenosis was $2.06 \%$ in patients undergoing surgical decompression over a 10-year time period, consistent with previous studies. Given the stringent inclusion and exclusion criteria in our study (surgical decompression of 2 regions within 1 year), it is likely that the incidence reported in our study
$(2.06 \%$ over 10 years) represents the lower end of the spectrum.

The diagnosis of tandem stenosis may be difficult. Almost half $(45 \%)$ of the patients in this series were diagnosed in a delayed fashion. A lack of clear recommendations for general screening for tandem stenosis may contribute to missed diagnoses. Myelopathic signs may not be present in up to $20 \%$ of patients. In our study, the delayed diagnosis of tandem stenosis was more likely to occur in patients presenting with lumbar symptoms without overt myelopathy. Conversely, patients undergoing cervical decompression initially were significantly more likely to have a diagnosis of tandem stenosis made preoperatively than made in a delayed fashion $(P=$ .014). While it is not cost effective to obtain a cervical/thoracic magnetic resonance imaging in every patient presenting with neurogenic claudication, a sagittal cervicothoracic screening sequence as suggested by Seo et $\mathrm{al}^{16}$ may reduce the risk of a delay in diagnosis. Failure to improve as expected following decompression surgery may indicate a tandem stenosis issue. Figure 2 demonstrates a diagnostic algorithm for evaluation of TSS.

Treatment for TSS has typically used staged decompression, specifically addressing the most symptomatic area of compression first. ${ }^{1,7,17}$ The prospect of single-stage surgery (either same day or simultaneous decompression) has been evaluated by several authors in a retrospective fashion, demonstrating good results. ${ }^{18}$ In 2011, Eskander et al ${ }^{4}$ evaluated the use of simultaneous versus staged decompression in a matched cohort study of 43 patients. Both groups demonstrated postoperative improvement in patient outcome scores (Oswestry Disability Index/Japanese Orthopaedic Association Score) with no difference noted at 7-year follow-up. Notably, an increase in minor complications was noted in patients over the age of 68 , procedures with a blood loss of more than $400 \mathrm{cc}$, or operative time of 150 minutes. $^{4}$ Krishnan et $\mathrm{al}^{19}$ followed 53 patients who underwent single-stage decompression and noted increased complications over the age of 60 and had recommended staged surgery in this population.

The authors recommend a careful approach to single-stage decompression, especially in the context of the elderly population, who appear to be the most affected. The authors generally recommend decompression of the cervical or thoracic region before the lumbar spine to protect the at-risk spinal cord from damage due to patient positioning. While patients 
who underwent initial lumbar decompression prior to cervical decompression did not demonstrate evidence of acute neurologic deterioration following lumbar decompression, some patients $(\mathrm{n}=3)$ developed upper extremity myelopathic symptoms. In addition to careful positioning, we routinely monitor upper extremity evoked potentials during lumbar decompression surgery to avoid potential positioning issues in the neck and upper extremities.

The study is limited by its retrospective nature. The goal of the study was to assess the incidence of tandem stenosis requiring surgical treatment of both regions. It is possible that some patients were lost to follow-up and underwent a second decompression at another institution, so our reported incidence may underestimate the true magnitude. In addition, we are unable to compare outcomes of patients surgically treated for tandem stenosis in comparison to patients with disease in only 1 region of the spine. However, based on our data, patients who have undiagnosed cervical stenosis may be at risk of neurologic deterioration when undergoing lumbar decompression.

\section{CONCLUSION}

TSS should remain within the differential for patients at initial presentation of spinal stenosis, especially lumbar spinal stenosis, as the symptoms of myelopathy may be difficult to detect in these patients. Catastrophic neurologic deterioration did not occur if lumbar stenosis was addressed prior to cervical stenosis, but new neurological symptoms may occur. Suspicion of tandem stenosis should be heightened if preoperative symptoms fail to expectedly improve following decompression even if overt myelopathic signs are not present, especially following lumbar decompression.

\section{REFERENCES}

1. Dagi TF, Tarkington MA, Leech JJ. Tandem lumbar and cervical spinal stenosis. Natural history, prognostic indices, and results after surgical decompression. $J$ Neurosurg. 1987;66(6):842-849.

2. Epstein NE, Epstein JA, Carras R, Murthy VS, Hyman RA. Coexisting cervical and lumbar spinal stenosis: diagnosis and management. Neurosurgery. 1984;15(4):489-496.

3. Molinari RW, Flanigan R, Yaseen Z. Tandem spinal stenosis (TSS): literature review and report of patients treated with simultaneous decompression. Curr Orthop Pract. 2012;23(4):356-363.

4. Eskander MS, Aubin ME, Drew JM, et al. Is there a difference between simultaneous or staged decompressions for combined cervical and lumbar stenosis? Clin Spine Surg. 2011;24(6):409-413.

5. Schaffer JC, Raudenbush BL, Molinari C, Molinari RW. Symptomatic triple-region spinal stenosis treated with simultaneous surgery: case report and review of the literature. Glob Spine J. 2015;5(6):513-521.

6. von Elm E, Altman DG, Egger $M$, et al. The Strengthening the Reporting of Observational Studies in Epidemiology (STROBE) statement: guidelines for reporting observational studies. Int J Surg. 2014;12(12):1495-1499.

7. Teng P, Papatheodorou C. Combined cervical and lumbar spondylosis. Arch Neurol. 1964;10(3):298-307.

8. Lee MJ, Garcia R, Cassinelli EH, Furey C, Riew KD. Tandem stenosis: a cadaveric study in osseous morphology. Spine J. 2008;8(6):1003-1006.

9. Bajwa NS, Toy JO, Ahn NU. Is congenital bony stenosis of the cervical spine associated with congenital bony stenosis of the thoracic spine? An anatomic study of 1072 human cadaveric specimens. Clin Spine Surg. 2013;26(1):E1-E5.

10. Bajwa NS, Toy JO, Young EY, Ahn NU. Is congenital bony stenosis of the cervical spine associated with lumbar spine stenosis? An anatomical study of 1072 human cadaveric specimens. J Neurosurg Spine. 2012;17(1):24-29.

11. Bajwa NS, Toy JO, Ahn NU. Is lumbar stenosis associated with thoracic stenosis? A study of 1,072 human cadaveric specimens. Spine J. 2012;12(12):1142-1146.

12. Park MS, Moon S-H, Kim T-H, et al. Asymptomatic stenosis in the cervical and thoracic spines of patients with symptomatic lumbar stenosis. Glob Spine J. 2015;5(5):366-371.

13. Park JY, Chin DK, Kim KS, Cho YE. Thoracic ligament ossification in patients with cervical ossification of the posterior longitudinal ligaments: tandem ossification in the cervical and thoracic spine. Spine (Phila Pa 1976). 2008;33(13):E407-E410.

14. Seo M, Choi D. Adjacent segment disease after fusion for cervical spondylosis; myth or reality? Brit J Neurosurg. 2008;22(2):195-199.

15. Adamova B, Bednarik J, Andrasinova T, et al. Does lumbar spinal stenosis increase the risk of spondylotic cervical spinal cord compression? Eur Spine J. 2015;24(12):2946-2953.

16. Seo J, Park SY, Lee JW, Lee GY, Kang HS. The value of additional cervicothoracic spine sagittal T2-weighted images included in routine lumbar spine MR imaging. J Korean Soc Magn Reson Med. 2013;17(2):91-100.

17. Aydogan M, Ozturk C, Mirzanli C, Karatoprak O, Tezer M, Hamzaoglu A. Treatment approach in tandem (concurrent) cervical and lumbar spinal stenosis. Acta Orthop Belg. 2007;73(2):234.

18. Kikuike K, Miyamoto K, Hosoe H, Shimizu K. Onestaged combined cervical and lumbar decompression for patients with tandem spinal stenosis on cervical and lumbar spine: analyses of clinical outcomes with minimum 3 years follow-up. Clin Spine Surg. 2009;22(8):593-601.

19. Krishnan A, Dave BR, Kambar AK, Ram H. Coexisting lumbar and cervical stenosis (tandem spinal stenosis): an infrequent presentation. Retrospective analysis of single-stage surgery (53 cases). Eur Spine J. 2014;23(1):64-73.

Corresponding Author: Daniel Gelb, MD, Department of Orthopaedics, University of Mary- 
land Medical Center, 110 South Paca Street, 6th Floor, Suite 300, Baltimore, MD 21201. Phone: (267) 229-5081; Fax: (410) 328-0534; Email: dgelb@, umoa.umm.edu.

Published 30 June 2019
This manuscript is generously published free of charge by ISASS, the International Society for the Advancement of Spine Surgery. Copyright (C) 2019 ISASS. To see more or order reprints or permissions, see http://ijssurgery.com. 